III. Aus Dr. I. Boas' Poliklinik für Magen- und Darmkrankheiten in Berlin.

\title{
Zur Frage der hämorrhagischen Erosionen des Magens.')
}

Von Dr. Hans Elsner, Assistenten der Poliklinik.

Wenn man den Magen eines Patienten in nüchternem $\mathrm{Zu}$ stande mit grösseren Mengen Wassers ausspült, so findet man im Spülwasser fast constant eine Anzahl zarter, grauweisslicher oder farbloser Gebilde. Dieselben bestehen, wie die mikroskopische Untersuchung lehrt, aus Convoluten von Schleim und Plattenepithelien, welch' letztere der Rachen- oder Oesophagusschleimhaut angehören, mit der Sonde in den Magen hinuntergestossen und mit dem Spülwasser wieder entfernt werden. Eine diagnostische Bedeutung kommt diesen Gebilden nicht zu.

In einer Reihe von Fällen findet man aber neben diesen farblosen Häutchen kleinere, fast immer blutig tingirte Gebilde, die sich durch ihre rothe Farbe von den erstgenannten scharf absetzen. Sie sind stecknadelkopf- bis über linsengross, haben unregelmässige, flottirende Ränder und zeigen unter dem Mikroskop die Struktur der oberflächlichsten Schichten der Magenschleimhaut, d. h. man sieht an ihnen gewöhnlich die Querschnitte der Vorräume von Magendrüsen mit dem normalen oder veränderten Epithel; das interstitielle Gewebe erscheint meist etwas vermehrt und ist regelmässig mit Blut infiltrirt.

Diese Schleimhautstückchen, die man ab und zu im Spülwasser des Magens findet, haben nach zwei Richtungen hin ein gewisses Interesse, in pathologisch-anatomischer und in klinischer Hinsicht. Die anatomische Seite dieser Frage, d. h. die Frage, ob und inwieweit die Mikroskopie dieser Schleimhautstückchen gestattet, Rückschlüsse zu ziehen auf etwaige pathologische Veränderungen der Magenschleimhaut - diese Frage ist in sehr zahlreichen Arbeiten, zum Theil sehr eingehend behandelt worden, in erster Reihe von Boas, ${ }^{2}$ ) ferner von Lubarsch, ${ }^{3}$ ) Hemmeter, ${ }^{4}$ ) Cohnheim, ${ }^{5}$ ) Leuk, ${ }^{6}$ Hari ${ }^{7}$ ) u. A., sodass es kaum noch möglich erscheint, neue Gesichtspunkte in dieser Richtung zu gewinnen. Die klinische Seite dieser Frage dagegen, die sich lediglich auf das Vorkommen der Schleimhautstückchen

1) Vortrag, gehalten im Verein für innere Medizin in Berlin, am 4. Mai 1903. - 2) Boas, Diagnostik und Therapie der Magenkrankheiten. 1894, S. 223. ${ }^{3)} \mathrm{Lub}$ arsch; Martius und Lubarsch, Die Achylia gastrica. 1897. 4) Hemmeter, Diseases of the stomach. ') Cohnheim, Festschrift für Prof. LazaruS 1899. 6) Leuk, Zeitschrift für klinische Medizin 1898, Bd. XXXVII. ) Hari. Archiv fitir mikroskopische Anatomie 1901. S. 685 . 
erstreckt, hat erst in den letzten Jahren ein gewisses Interesse erlangt, und zwar dadurch, dass Einhorn ${ }^{1}$ ) auf dem Vorkommen dieser Schleimhautstückchen im Spülwasser des nüchternen Magens ein Krankheitsbild aufbaute, welches er mit dem Namen „hämorrhagische Erosionen des Magens" belegte.

Nach den Beschreibungen, welche bisher von diesem Krankheitsbild gegeben worden sind, ist dasselbe dadurch charakterisirt, dass sich neben den meist, aber nicht immer vorhandenen Zeichen einer mehr oder minder ausgesprochenen Gastritis, resp. Achylia gastrica, kleine Erosionen oder Exfoliationen in der Magenschleimhaut finden, die sich klinisch durch den Abgang solcher Schleimhautstückchen bei der Bespülung des Magens dokumentiren. Wenn man also bei einem Patienten mit einer chronischen Gastritis oder mit einer Achylia gastrica Spülungen des nüchternen Magens vornimmt und dabei constant - darauf legen einzelne Autoren den Nachdruck - solche Schleimhautstückchen im Spülwasser findet, dann sollen solche „hämorrhagische Erosionen" im Magen vorhanden sein, allerdings nur dann, wenn auch subjektiv gewisse Erscheinungen bestehen, welche auf das Vorhandensein der Schleimhautstückchen hindeuten. Der objektive Befund bei den bisher beschriebenen Fällen von „hämorrhagischen Erosionen" lässt sich also in zwei Worten zusammenfassen: Entzündung der Magenschleimhaut und Erosionen, resp. Exfoliationen.

Dieser Zusammenhang zwischen chronischer Entzündung der Magenschleimhaut und Geschwürsbildungen derselben ist ein altbekannter.

Schon im Jahre 1853 hat $\mathrm{Virchow}^{2}$ ) darauf hingewiesen, dass akute und chronische Katarrhe, namentlich solche, welche mit starken krampfhaften Zusammenschnïrungen des Magens verbunden sind, zu hämorrhagischen Erosionen und blutenden Geschwüren führen können. Später hat Nauwerk" ${ }^{3}$ ) die grundlegende Arbeit für diese Frage geliefert, indem er einen Sektionsbefund veröffentlichte, bei dem sich in einer diffus entzündeten Magenschleimhaut zahlreiche Erosionen und tiefergehende Defekte fanden. Nauwerk glaubt, dass Gastritis und Erosionen, resp. die aus ihnen hervorgehenden Ulcerationen einen einheitlichen Krankheitsvorgang entzündlicher Natur darstellen. "Erosion und die entsprechende Form der Ulceration sind nach $\mathrm{Nauwerk}$ Theilerscheinungen einer chronischen Gastritis. Eine ähnlich lautende Angabe macht Mathieu,,$^{4}$ ) welcher gleichfalls die Beobachtung machte, dass sich bei der chronischen Gastritis entweder einfache folliculäre Erosionen oder Sogar Ulcerationen ausbilden können. Nach beiden A.utoren sind die Ulcerationen, die man bei Gastritis findet, und die gewöhnlich aus Erosionen hervorgegangen sind, scharf zu trennen von dem gewöhnlichen Ulcus ventriculi rotundum, bei welchem die chronische Gastritis meist fehlt oder, wo sie vorhanden, doch nicht als Ursache desselben anzusprechen ist. - Aehnliche Sektionsbefunde, in welchen sich chronische Grastritis in Verbindung mit ausgedehnten Erosionen und Ulcerationen der Schleimhaut fand, sind später von Langerhans, $\left.{ }^{5}\right)$ D. Gerhardt ${ }^{6}$ ) u. A. veröfentlicht worden. Langerhans sagt auf Grund seines Sektionsbefundes: „Gewöhnlich entstehen hämorrhagische Erosionen bei krampfhaften Contraktionen der Magenwand und gleichzeitigen entzündlichen Prozessen."

Einen sehr wichtigen Beitrag zu dieser Frage hat noch Dieulaf o ${ }^{7}$ ) geliefert, welcher einer bestimmten Form der hämorrhagischen Erosionen in seinen Abhandlungen über innere Medizin ein besonderes Kapitel widmet. Dieulaf oy beschreibt u. a. die Krankengeschichte von zwei Patienten, die mit einer Pneumonie ins Krankenhaus aufgenommen wurden und nach wenigen Tagen profuse Magenblutungen bekamen, an denen der eine auch zu Grunde ging. Bei der Autopsie fand sich in dem einen Fall eine Allgemeininfektion mit Pneumococcen; die Magenschleimhant war diffus entzündet und mit zahlreichen kleinen Erosionen bedeckt. Di eulaf oy führt diesen lokalen Befund am Magen auf die Wirkung der Pneumococcen zurück und bezeichnet demgemäss diese Affektion mit dem Namen: Gastrite ulcéreuse hémorrhagique pneumococcique. - Obwohl diese Sektionsbefunde von Dieulafoy ein Krankheitsgenus für sich bilden, schon wegen ihres infektiösen Charakters, so zeigen sie doch ebenfalls, wie Entzuindung und Ulceration als einheitliche Krankheitsprozesse Hand in Hand gehen können.

Während aus alledem hervorgeht, dass schon vor 15 und 20 Jahren Sektionsbefunde von "hämorrhagischen Erosionen“" vorlagen, war über das klinische Bild der Krankheit nichts bekannt, bis, wie schon erwähnt, Einhorn im Jahre 1895 einen

1) Einhorn, Berliner klinische Wochenschrift 1895, No. 20. 2) Virchow, Virchow-Archiv, Bd. V, S. 363. - ${ }^{9}$ ) Nauwe rk, Münchener medizinische Wochenschrift 1897. - *) Mathieu, Traité de Médecine, 1892. - ${ }^{5}$ ) Langerhans, Virchow-Archiv, Bd. CXXIV, S. 373. 8) D. Gerhardt, Virchow-Archiv, Bd. CXXVII, S. 85. - 7) Di eulafoy, Manuel de Pathologie interne, 1901, S. 263.
Symptomencomplex beschrieb, dem er den Namen „hämorrhagische Erosionen" gab.

Ich möchte mir erlauben, dieses Krankheitsbild kurz zu skizziren an der Hand eines Falles, den ich in der Poliklinik meines verehrten Chefs, des Herrn Dr. Boas, zu beobachten Gelegenheit hatte:

Ein 45jähriger Postschaffner B., der erblich nicht belastet ist, giebt an, vor 20 Jahren einen Typhus durchgemacht zu haben, sonst aber niemals ernstlich krank gewesen zu sein. Vor einem Jahre hatte er zum ersten Male Magenbeschwerden; er wurde damals an chronischem Magenkatarrh behandelt. Seitdem arbeitete er längere Zeit mit wechselndem Befinden. Vor drei Monaten stellten sich wiederum Magenbeschwerden ein, und zwar hauptsächlich Magenschmerzen. Durch ärztliche Behandlung erfolgte keine Besserung; der Patient nahm im Verlaufe einiger Wochen Sieben Pfund an Gewicht ab. Er klagt jetzt über dauernden Druck in der Magengegend, der auch bei leerem Magen besteht. Durch Nahrungsaufnahme treten regelmässig brennende Schmerzen im Magen auf, die nicht sehr heftig sind, aber doch constant nach fester Nahrung sich einstelien. Flüssige Sachen verträgt Patient besser. Einige Male trat Erbrechen auf, in der letzten Zeit jedoch nicht mehr. Der Stuhilgang ist regelmässig.

Aus dem Status möchte ich nur den Befund am Magen hervorheben: Die funktionelle Untersuchung des Magens ergab folgenden Befund: Ein Probefrühstück, welches Patient erhielt, wurde sehr schlecht verdaut, der Rückstand wies grossen Schleimgehalt auf; dabei war die Salzsäure- und Fermentabscheidung wenig herabgesetzt. Jedenfalls musste auf Grund des Befundes ein gastritischer Prozess angenommen werden. - Als der Patient am nächsten Tage nüchtern ausgehebert wurde, wurden cirka $10 \mathrm{ccm}$ einer neutral reagirenden, Schleim enthaltenden Flüssigkeit entleert. In dieser wurde ein über linsengrosses Schleimhautstückchen gefunden. Daraufhin wurde dem Patienten nüchtern der Magen zu wiederholten Malen ausgespült. Bei den ersten Spülungen fanden sich im Spülwasser regelmässig zahlreiche Schleimhautstückchen von verschiedener Grösse, bei einer nach acht Tagen vorgenommenen Spülung schon nicht mehr, sodass der Patient ohne Beschwerden entlassen werden konnte.

Diese Krankengeschichte entspricht ungefähr den Fällen, die Einhorn veröffentlicht hat. Es handelt sich um einen Patienten mit Erscheinungen von Gastritis, die ganz alltägliche Beschwerden hervorruft. Auffallend sind die Schmerzen, über die der Patient klagt, die ja sonst bei Gastritis nicht gerade gewöhnlich sind. Da diese Schmerzen im Anschluss an die Nahrungsaufnahme auftreten, so werden wir vielleicht nicht fehlgehen, wenn wir sie als eine Folge der kleinen Schleimhautexfoliationen betrachten, wie das auch Einhorn für seine Fälle angiebt. In Folge der Schmerzen ist die Nahrungsaufnahme herabgesetzt, und der Patient nimmt an Körpergewicht ab. Schmerzen, Schwächegefühl und Abmagerung sollen also die subjektiven Symptome sein, welche die hämorrhagischen Erosionen charakterisiren.

Nun sind nach dem Vorbilde von Einhorn eine ganze Reihe analoger Krankengeschichten veröffentlicht worden, so von Sansonil), Pariser ${ }^{2}$ ), Mintz ${ }^{3}$ ) u. A., bisher konnte aber noch keine dieser klinischen Beobachtungen durch eine Sektion erhärtet werden, d. h. es existirt noch kein Sektionsbefund, durch den der Nachweis erbracht worden wäre, dass da, wo intra vitam die genannten klinischen Erscheinungen bestanden, auch wirklich hämorrhagische Erosionen am Magen vorhanden waren.

Weiterhin lässt sich gegen diese Beobachtungen einwenden, dass noch keine Untersuchungen darüber angestellt sind, ob die Schleimhautstückchen wirklich einen so charakteristischen Befund darstellen, dass man sie zur Grundlage eines neuen Krankheitsbildes machen könnte, ob bestimmte Magenaffektionen, und welche, zu solchen Abschilferungsprozessen neigen.

Dieser letzteren Frage, auf der sich eigentlich das ganze Gebäude der hämorrhagischen Erosionen erhebt, habe ich näherzutreten versucht, indem ich in der Poliklinik des Herrn Dr. Boas Patienten mit den verschiedensten Magenaffektionen auf das Vorkommen von solchen Schleimhautstückchen untersuchte. Mir stehen in dieser Richtung etwa 120 Fälle zur Verfügung. Auf Grund dieses Materials kann ich sagen, dass man in einem ganz gesunden Magen die Schleimhautstückchen niemals findet; einen pathologischen Befund stellen dieselben also sicherlich dar. Im übrigen muss man unterscheiden, ob entzündliche Erscheinungen am Magen mit Herabsetzung oder Aufhebung der Sekretion nachweisbar, oder ob keine sichtbaren Erscheinungen entzündlicher

1) Sansoni, Archiv für Verdauungskrankheiten, Bd. VI, Heft 1 2) Pariser, Berliner klinische Wochenschrift 1900. - 3) $\mathrm{Mintz}$ Zeitschrift für klinische Medizin 1902. 
Natur vorhanden sind. Bei dieser letzteren Gruppe habe ich nur einige wenige Male die Schleimhautstückchen gefunden, vorzugsweise bei Hyperacidität, einige Male auch bei Ektasie des Magens mit erhöhter Salzsäureproduktion.

Was die erstgenannte Gruppe anbetrifft, so habe ich im ganzen 35 Fälle untersucht, bei denen mehr oder minder erhebliche Sekretionsstörungen vorlagen. Unter diesen habe ich $12 \mathrm{mal}$ vorübergehend oder dauernd Schleimhautstückchen im Spülwasser gefunden. Daraus geht hervor, dass solche exfoliirenden Prozesse der Magenschleimhaut, welche zu dem Auftreten von Schleimhautstückchen im Spülwasser Veranlassung geben, beiGastritis chronica, resp. bei Achylia gastrica ziemlich häufig (etwa in $35 \%$ der Fälle) auftreten, während sie bei anderen Magenaffektionen ziemlich selten sind. Dieser Befund ist bereits bekannt. Ewald ${ }^{1}$ ) erwähnt ihn bei Besprechung der Gastritis mucosa, auch Strauss ${ }^{2}$ ) hat ihn in einigen Fällen von Dyspepsia gastrica, Boas ${ }^{3}$ ) in Fällen von chronischer Gastritis erhoben; letzterer fand die Schleimhautstückchen auch in Fällen von Superacidität und Neurosen des Magens.

Es hat sich nun gezeigt, dass die Schleimhautstückchen, die ich in den genannten 12 Fällen gefunden habe, in der Mehrzahl dieser Fälle überhaupt keine klinischen Erscheinungen hervorriefen. In diesen Fällen sind also diese kleinen Abschilferungen offenbar nur ein nebensächlicher Befund, und da es sich hier um die Mehrzahl der Fälle handelt, so wird man sagen dürfen, dass in der Mehrzahl der Fälle der Befund von Schleimhautstückchen bei entzündlichen Zuständen der Magenschleimhaut nur eine untergeordnete klinische Bedeutung besitzt. Aus diesem Grunde erscheint es auch nicht empfehlenswerth, das Vorkommen von diesen Schleimhautstückchen zur Grundlage eines neuen Krankheitsbildes zu machen und dieselben als charakteristisch für dieses Krankheitsbild zu bezeichnen. Das sind sie keineswegs.

Nur in 4 von den genannten 12 Fällen klagten die Patienten über Magenschmerzen, und nur in 3 von diesen Fällen traten die Schmerzen in charakteristischer Weise auf, sodass man eigentlich nur diese drei Fälle als hämorrhagische Erosionen im Sinne Einhorn's auffassen kann. Der objektive Befund in diesen drei Fällen zeigt, abgesehen von den kleinen Erosionen, nichts Charakteristisches. Bezüglich der sekretorischen und motorischen Magenfunktion finden sich dieselben Störungen, die man auch sonst bei Gastritis, resp. bei Achylia gastrica findet; auch die Schleimbildung kann gering sein oder exzessive Grade erreichen.

Was dagegen diese Fälle von anderen Formen der Gastritis unterscheidet, das sind nur die subjektiven Symptome, in erster Reihe die Schmerzen, die allerdings in den genannten drei Fällen nicht sehr heftig sind. Gleichwohl ist die Möglichkeit nicht von der Hand $\mathrm{zu}$ weisen, dass dieselben gelegentlich einmal so stark werden können, dass ihnen gegenüber die eigentlichen gastritischen Erscheinungen in den Hintergrund treten. In der Art ihres Auftretens erinnern die Schmerzen an einen ulcerösen Prozess der Schleimhaut, da sie in ganz charakteristischer Weise im Anschluss an die Nahrungsaufnahme auftreten. Da sie in meinen Fällen nicht sehr heftig waren, so war auch eine irgendwie erhebliche Abmagerung nicht $\mathrm{zu}$ constatiren. Die Schmerzen hören gewöhnlich auf mit dem Verschwinden der Schleimhautstückchen, doch zeigen sie manchmal Neigung, zu rezidiviren. Es kommt vor, dass Patienten, die durch geeignete Diät von den Erosionen befreit werden, schon kurze Zeit nachher mit ihren Beschwerden wiederkommen. Sonstige klinische Folgeerscheinungen der kleinen Schleimhautexfoliationen habe ich nicht beobachten können; in einem Falle waren Magenblutungen vorhergegangen, wie sie bei folliculären Erosionen des Magens, die durch die Sektion erhärtet sind, beobachtet wurden.

Wenn man diese Fälle, die ja nur eine besondere Form der Gastritis darstellen, mit den von Einhorn beschriebenen Fällen vergleicht, so ergiebt sich eine völlige Uebereinstimmung der objektiven und subjektiven Symptome. Daher wird sich vielleicht die Annahme rechtfertigen lassen, dass sich aus einem entzündlichen Zustand des Magens, wenn die Neigung zu solchen Abschilferungsprozessen in der Sohleimhaut besteht, leicht das erwähnte Krankheitsbild entwiokeln kann. Die ursprünglich allein

1) Ewald, Eulenburg's Realencyklopädie, Bd. XIV, S. 295. 3) Strauss, Zeitschrift für klinische Medizin, Bd. XLI, S. 281. 3) Boas, l. c. vorhanden gewesene Gastritis wird dann mehr und mehr verdeckt werden durch die Erscheinungen, welche von Seiten der Erosionen ausgehen.

$\mathrm{Ob}$ nun aber diesem Symptomenbild wirklich solche anatomischen Veränderungen zu Grunde liegen, wie sie von Nauwerk, Gerhardt und Langerhans beschrieben worden sind, das muss mangels irgend eines Sektionsbefundes zum mindesten noch zweifelhaft erscheinen. Wenn man nämlich diese Fälle mit den vorliegenden Sektionsbefunden vergleicht, so ergiebt sich eine auffallende Verschiedenheit. Während bei den Sektionsbefunden die am Magen vorgefundenen Veränderungen zumeist sehr schwerer und ausgedehnter Natur waren, zeigen die unter demselben Namen (von Einhorn) publizirten klinischen Fälle Erscheinungen, die zumeist leichter Natur sind und eine ausgesprochene Tendenz zur Heilung aufweisen. Aus diesem Grunde wird es sich empfehlen, den Namen "hämorrhagische Erosionen" für dieses Krankheitsbild so lange $z u$ vermeiden, als nicht durch eine Sektion der Nachweis erbracht ist, dass diese Fälle ihrem Wesen nach wirklich identisch sind mit den an der Leiche festgestellten Befunden von hämorrhagischen Erosionen. Vorläufig spricht allerdings sehr viel gegen, sehr wenig für eine solche Identität; umsomehr ist die Vorsicht anzuerkennen, mit der sich Einhorn hinsichtlich seiner Fälle ausgedrückt hat.

Zur Zeit lässt sich eben nur soviel sagen, dass in manchen Fällen von entzündlichen Sekretionsstörungen, in denen man im Spülwasser des Magens die Schleimhautstückchen findet, gleichzeitig auch subjektiv gewisse Symptome, in erster Reihe Schmerzen auftreten, welche auf das Vorhandensein der kleinen Exfoliationen hindeuten. Obwohl diese Fälle nicht gerade häufig sind, so lehren sie doch, dass man gut thun wird, in allen Fällen von chronischer Gastritis, in denen über solche Schmerzen in der Magengegend geklagt wird, an Abschilferungsprozesse der Schleimhaut zu denken und Spülungen des nüchternen Magens vorzunehmen. Die Beurtheilung des Falles wird dadurch nur wenig geändert, für die Therapie bleibt der Befund von Schleimhautstückchen im Spülwasser in der Mehrzahl der Fälle vollends bedeutungslos. Denn in allen Fällen genügte die diätetische Behandlung, welche die Gastritis an sich erforderte, um die Schleimhautstückchen zum Verschwinden zu bringen. In einem Fall, bei einer 40jährigen Frau, habe ich, wie das von Einhorn empfohlen ist, Magenspülungen mit Argentum nitricumLösung gemacht, und zwar mit gutem Erfolg.

Selbst wenn man auf dem Standpunkt steht, dass die hämorrhagischen Erosionen des Magens als klinisches Krankheitsbild eine gewisse Existenzberechtigung haben - ich persönlich halte das, wie erwähnt, für unwahrscheinlich - , so wird man sie doch nicht als selbständige Erkrankung, sondern höchstens als eine etwas abweichende Form einer Gastritis auffassen können. In den einschlägigen Lehrbüchern werden diese Fälle daher ihren Platz haben müssen als ein Änhang zum Kapitel „Gastritis chronica“. 